Cita bibliográfica: Prat Forga, J. M. (2020). La puesta en valor turístico del patrimonio cultural en fase de desaparición. Una oportunidad para los territorios de interior. Los casos de los monasterios de Vilabertrán, Scala Dei y Escornalbou, en Catalunya. Investigaciones Turísticas (20), pp. 315-334. https://doi.org/10.14198/INTURI2020.20.14

\title{
La puesta en valor turístico del patrimonio cultural en fase de desaparición. Una oportunidad para los territorios de interior. Los casos de los monasterios de Vilabertrán, Scala Dei y Escornalbou, en Catalunya
}

The enhacement of cultural heritage in phase of disappearance. An opportunity for inland territories. The cases of the monasteries of Vilabertrán, Scala Dei and Escornalbou, in Catalonia

Josep Maria Prat Forga (iD, Universitat Autònoma de Barcelona, España impratf@gmail.com

\section{RESUMEN}

Los territorios de interior que poseen un importante patrimonio cultural en fase de destrucción tienen la oportunidad de restaurarlo y rehabilitarlo, poniéndolo en valor turístico. De esta manera, no solamente reafirman el sentimiento de identidad local y promocionan su cultura sino que también ayudan a su desarrollo económico, recibiendo nuevos visitantes, ávidos de consumir cultura e historia, y que, no solamente redescubren estos conjuntos monumentales, habiéndose informado previamente de las principales características de la visita, sino que, además, efectúan otros gastos adicionales en sus alrededores, lo que permite crear nuevos puestos de trabajo y mejorar la renta personal de la comunidad local.

En este trabajo se analiza la evolución de la cantidad anual de visitantes recibidos en los últimos quince años en tres complejos religiosos que sufrieron su abandono y destrucción a partir de la desamortización de Mendizábal, y cuya restauración y puesta a punto ha permitido situarlos dentro del circuito turístico y cultural de los monasterios catalanes.

Para ello se ha utilizado una serie de datos estadísticos oficiales y descriptivos; así como los resultados de una encuesta personal realizada al azar a los visitantes de estos monumentos, confirmando que manifiestan un alto grado de satisfacción con la visita y que están dispuestos a repetirla 0 , al menos, promocionarla.

Palabras clave: turismo cultural, patrimonio, monasterios, satisfacción, Catalunya.

\section{ABSTRACT}

Inland territories with an important cultural heritage but which are in the process of deterioration have the opportunity to restore and rehabilitate this heritage, enhancing its tourism 
value. In this way, not only do they reinforce the feeling of local identity and promote their culture but also help their economic development, receiving new visitors, eager to consume culture and history. As well as rediscovering these monumental groups, having previously been informed of the main characteristics of the visit, these visitors also make other additional expenditures in the surrounding areas. This creates new jobs and improves the personal income of the local community.

This study analyses the evolution of the annual number of visitors received over the last fifteen years in three religious complexes that had been abandoned and destroyed after the Mendizábal disentailment law. Their restoration has positioned them within the tourist and cultural circuit of the Catalan monasteries. A series of official and descriptive statistical data has been used for the analysis, together with the results of a random personal survey of visitors to these monuments. The results of this survey confirm a high degree of satisfaction with the visit and the willingness of the visitors to return or at least promote it

Keywords: cultural tourism, heritage, monasteries, satisfaction, Catalonia.

\section{INTRODUCCIÓN Y OBJETIVOS}

En la actualidad nos encontramos, por una parte, ante un mundo globalizado, en el que el dinero, las comunicaciones, los transportes y la información han rebasado las fronteras y circulan de manera instantánea y donde la gente, ávida de conocimiento, observa y reflexiona sobre todo lo que le rodea, interpretándolo (Urry, 1990). Por la otra, los turistas han modificado sustancialmente sus hábitos de vida, sus motivaciones y sus expectativas, por lo que se han producido profundos cambios en el proceso de selección de un destino donde viajar, demandando unas actividades donde la experiencia, la personalización y la calidad son fundamentales (Urry, 1990; Goeldner, Ritche y McIntosh, 2000; Farrell y Twining-Ward, 2004; Martínez, 2006, 2015; Pearce, 2012; Prat, 2012, 2013; Prat y Cànoves, 2014; Williams y Lew, 2015; Richards, 2017).

Ante esta situación, en las últimas décadas se está constatando cómo el turismo va ampliando sus modalidades y productos con el objetivo de satisfacer estas nuevas tendencias. Este fenómeno ha sido muy marcado en el caso del turismo cultural, el cual ha modificado sustancialmente su enfoque y sus planteamientos, ya que está orientado a un colectivo que, cada vez con mayor intensidad, desea conocer y relacionarse con la historia y la cultura de los territorios que se visitan (Smith y Richards, 2012; Torres, 2015), consolidándose como una práctica social que ha provocado una culturización de la sociedad occidental, produciéndose un solapamiento entre turismo y cultura (Lyon, 1996; Rojek y Urry, 1997; Lyotard, 2000; Molina, 2006).

Así pues, una de las características básicas de este turismo cultural posmoderno es la uniformización de los fenómenos sociales, culturales y económicos, teniendo en cuenta el nuevo perfil de este tipo de turista, que, como consumidor selectivo, autogestiona sus actividades de ocio y recreación con ayuda de las nuevas tecnologías, especialmente internet (Blanco y Genovés, 2005; Richards, 2011, 2017; Donaire, 2012). De esta forma, el turismo cultural se ha convertido en un turismo creativo, donde la mayor cultura digital y el aumento de 
las atracciones culturales han provocado un crecimiento de las experiencias turísticas activas (Richards y Raymond, 2000; Richards, 2011; Hatch y Cunliffe, 2013).

Desde esta perspectiva, la creatividad no solamente es un requerimiento del turista cultural sino también del propio destino, produciéndose cambios en los roles de todos los agentes implicados y en el significado de las prácticas turísticas, lo que ha puesto de manifiesto la necesidad de disponer de unas adecuadas redes locales de conocimiento y creatividad (Smith y Richards, 2012; Torres, 2015; Richards, 2017). Por ello, el turismo cultural se ha convertido en una manera de activar o destacar elementos y manifestaciones que podrían ser considerados como patrimonio de un determinado territorio, transformándolos en atractivos turísticos (Molina, 2006; Martínez, 2015; Richards, 2017).

En este contexto, los territorios de interior, cuyo desarrollo económico se basaba fundamentalmente en el sector primario, están impulsando nuevas actividades terciarias que generen beneficios a estas zonas, tanto directos (como los pagos que realizan los turistas) como indirectos (por ejemplo, las compras de bienes y servicios) e inducidos (como las compras por parte de los proveedores de bienes y servicios para el propio consumo) (Wall y Mathieson, 2006; Hudson, 2010; Cànoves et al., 2014).

Además, se trata de una actividad que ayuda a diversificar el desarrollo económico y social de estos destinos, contribuyendo a generar nuevos negocios y puestos de trabajo (Prat y Cànoves, 2014), mientras que en el aspecto socio-cultural es una oportunidad de intercambio cultural y formación mutua entre los vecinos y los visitantes, lo que afianza la identidad local y ayuda a preservar la autenticidad de las tradiciones y la diversidad (Hu y Ritchie, 1993; Hall, Gössing y Scott, 2015).

En ocasiones, la transformación de estos territorios ha desembocado en la urbanización del espacio rural (Fløysand y Jakobsen, 2007), desarrollándose alrededor del turismo cultural múltiples actividades sustitutivas o complementarias a las más tradicionales (Scott, Baggio y Cooper, 2008; López Palomeque y Cànoves, 2014). No obstante, en muchas ocasiones, el desarrollo de este tipo de turismo en estas áreas rurales no ha implicado la readaptación del territorio a las nuevas exigencias de los turistas (Pearce, 2012; Cànoves et al., 2014).

Sea como sea, muchos territorios de interior que poseen un determinado patrimonio cultural hacen importantes esfuerzos para rehabilitarlo y ponerlo en valor turístico, apoyados en la colaboración de los agentes locales, la población autóctona, el entorno natural, la cada vez mayor concienciación medioambiental y los planes de inversión pública en estos municipios (Walker y Salt, 2006; Prat, 2013; García Hernández, 2014). Sin embargo, la reciente crisis económica, iniciada en los años 2007-2008, también ha afectado a esta actividad turística, ya que se ha reducido el poder adquisitivo de la población, en general, lo que ha llevado a un menor consumo, especialmente entre la clase media, provocando una disminución de los desplazamientos de ocio y del gasto de estos visitantes en estos territorios (Pearce, 2012).

Ante esta situación, el presente artículo analiza la evolución turística de tres conjuntos monumentales catalanes, a lo largo de estos últimos quince años, que fueron afectados gravemente por la desamortización de Mendizábal de 1835 y que, en la actualidad, forman parte del patrimonio cultural de Catalunya (Figura 1). En concreto, se trata del monasterio cartujo 
de Scala Dei y del monasterio-castillo de Sant Miquel d'Escornalbou, ambos en la provincia de Tarragona, y de la colegiata-monasterio de Santa María de Vilabertrán, en la provincia de Girona.

El principal objetivo de este trabajo es conocer el perfil socio-demográfico de los actuales visitantes a estos recintos, cuál es su fuente previa de información antes de desplazarse a estos destinos, cuáles han sido sus motivaciones para realizar el viaje y que factores más han apreciado una vez realizada la visita; así como su grado de satisfacción con la misma.

Con dicho análisis se ha podido constatar que la reciente crisis económica no ha afectado en demasía al flujo de visitantes recibidos en estos tres monumentos, que los visitantes se han informado previamente sobre el destino que van a visitar y sobre su patrimonio y sus principales monumentos, que para recibir esta información previa se han utilizado preferentemente las herramientas disponibles en internet y que se ha alcanzado un alto grado de satisfacción con la visita, repitiéndola y/o prescribiéndola a otros futuros visitantes.

También se han analizado las razones por las que estos visitantes han efectuado el desplazamiento y han consumido turismo cultural, confirmándose que su principal motivación era pasar una jornada vacacional, generalmente en familia o con amigos, aprovechando para conocer más estos territorios, su paisaje, cultura y sus monumentos más emblemáticos.

Figura 1. Mapa de situación de los conjuntos monumentales analizados

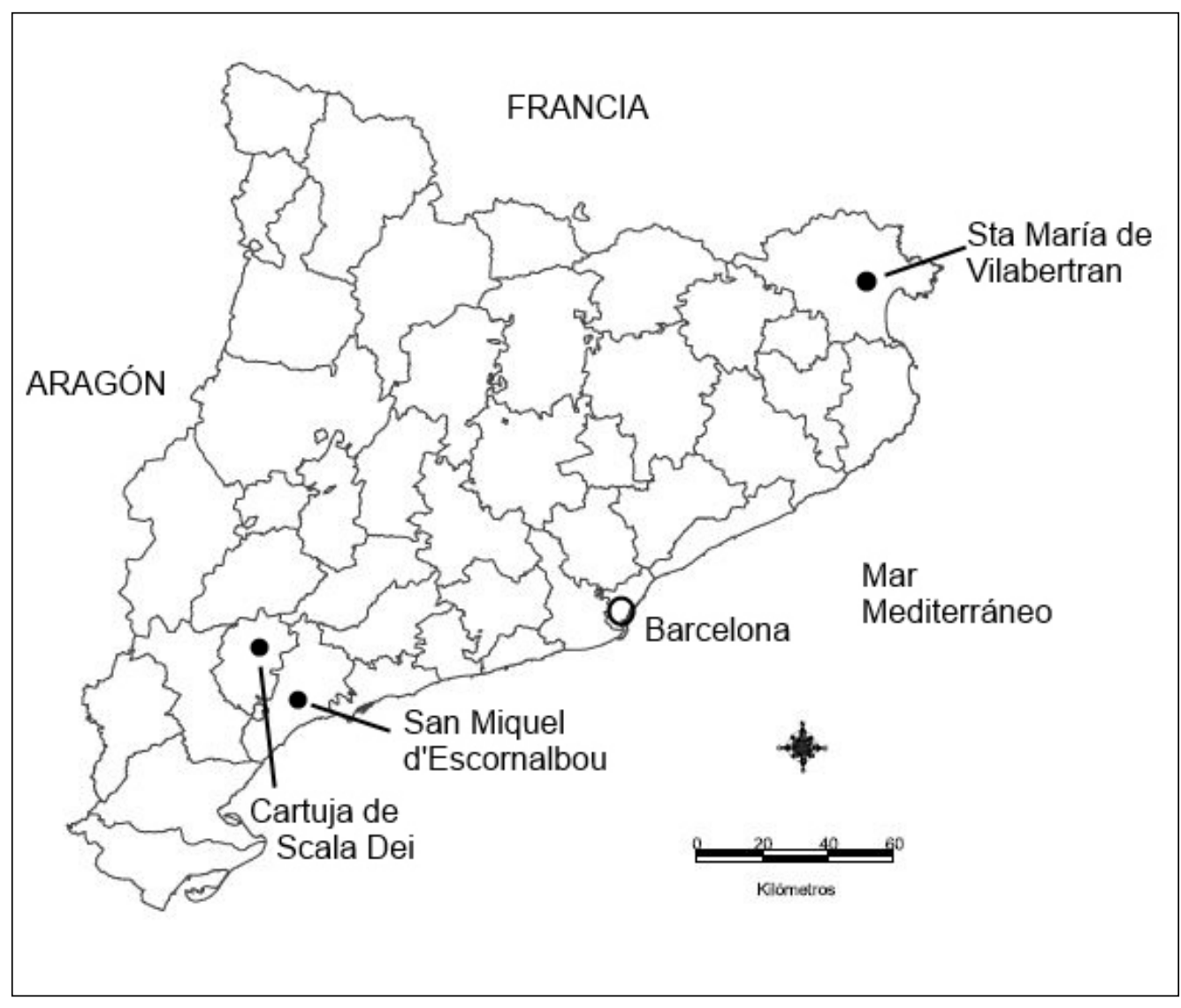

Elaboración propia con gvSIG. 


\section{LA REVALORIZACIÓN TURÍSTICA DEL PATRIMONIO CULTURAL}

Durante el siglo XX se ha producido una continua evolución en el concepto de patrimonio, en los elementos que lo componen y en sus usos (Rivera y Fernández-Baca, 2004), lo que demuestra la importancia de estos bienes, así como la necesidad de su rehabilitación, restauración, conservación y mantenimiento. Estos cambios, que contemplan aspectos tangibles e intangibles, permiten poner en valor su esencia de identificador de una determinada sociedad y elemento cohesionador del entorno territorial (Mínguez, 2007).

De esta forma, el concepto de patrimonio se ha ido ampliando con el tiempo, pasando de una visión restrictiva, histórico-artística, a otro con una mayor presencia de la componente social y territorial, pudiendo hablarse de su "territorialización" (Di Meo, 1995). Así pues, la valoración del territorio como patrimonio no solamente ha obedecido a la evolución conceptual del patrimonio, sino también del territorio, el cual se ha convertido en un recurso cultural (Mínguez, 2007).

Asimismo, la utilidad del patrimonio también se ha modificado, pasando de tener una vinculación directa con la posesión de unos bienes, el prestigio social y el poder, a una visión más basada en la comprensión y transmisión del conocimiento del pasado, lo que implica la conservación y conocimiento de los bienes, y su interpretación, difusión y puesta en valor (Peral, 1999).

En consecuencia, los territorios que poseen un patrimonio cultural de una cierta relevancia suelen realizar un importante esfuerzo económico, con ayudas de las administraciones públicas superiores, para rehabilitarlo y ponerlo en valor turístico, dotándolo de unos accesos y servicios adecuados, proveyéndolo de personal de información y de material promocional y difundiéndolo a través de las redes sociales (Hudson, 2010; Hall, Gossing y Scott, 2015).

Por su parte, el turismo cultural también ha sufrido importantes cambios, motivados por la rápida evolución que han experimentado los intereses y las motivaciones de unos turistas, cada vez más ávidos de consumir cultura; así como la voluntad de las administraciones públicas por poner en valor los recursos patrimoniales del territorio, lo que ha provocado la puesta en valor turístico de muchos de ellos (Mínguez, 2007).

Análogamente, las definiciones de turismo cultural se han venido sucediendo a lo largo de las últimas décadas (Smith, 2015). Así, en 1985, la Organización Mundial del Turismo (OMT) lo definía como "el movimiento de personas debido esencialmente a motivos culturales, como viajes de estudios, viajes a festivales $u$ otros eventos artísticos, visitas a sitios y monumentos, viajes para estudiar la naturaleza, el arte, el folklore, y las peregrinaciones para satisfacer la humana necesidad de la diversidad, orientados a elevar el nivel cultural del individuo, facilitando nuevos conocimientos, experiencias y encuentros". Así pues, con esta definición, el turista no se limitaba sólo a adoptar una actitud contemplativa, sino que, con el viaje, buscaba realizar una experiencia.

Posteriormente, en 1995, Silberberg lo definió como aquellos desplazamientos realizados, fuera de la residencia habitual, motivados por el interés en los aspectos históricos y 
culturales de una determinada comunidad (Silberberg, 1995). A su vez, en 1996, Richards lo presentó como el desplazamiento temporal de unas personas motivadas por conocer la historia y la cultura de un territorio, así como consumir nuevas experiencias que diesen satisfacción a las necesidades culturales personales de estos visitantes (Hu y Ritchie, 1993; Richards, 1996; Farrell y Twining-Ward, 2004).

Más recientemente, en 2015, Du Cros y McKercher indicaron que el turismo cultural ayudaba a poner en valor el patrimonio de los destinos, transformándolo en productos susceptibles de ser consumidos por los turistas (Du Cros y McKercher, 2015). Sin embargo, esta definición no consideraba la situación actual de la comunidad visitada, por lo que Smith concluyó que el turismo cultural era una actividad de ocio y recreación que estaba muy relacionada con el patrimonio material e inmaterial de una determinada comunidad, de forma que el visitante se beneficiaba, a la vez, del consumo de nuevas experiencias formativas, creativas y recreativas (Smith, 2015).

El turismo cultural comprende una gran diversidad de tipologías y no todos los turistas culturales manifiestan el mismo grado de interés por la cultura, de forma que algunos autores sugieren que la mayor parte de este tipo de turistas son bastante casuales, no siendo el consumo de la cultura local la motivación principal de su viaje (Martos y Pulido, 2010; McKercher, 2002; Du Cros y McKercher, 2015; Smith, 2015). Por ello, Silberberg y Hughes propusieron, por separado y en diferentes momentos, una clasificación de los turistas culturales según sus motivaciones personales.

Dicha clasificación es: a) los que están fuertemente motivados por los aspectos culturales del viaje, de manera que lo realizan fundamentalmente para satisfacer esta motivación; b) los que tiene una motivación cultural parcial para realizar el viaje, siendo otro el motivo principal del desplazamiento; c) los que no para tienen una motivación cultural para realizar el viaje, pero que aprovechan su estancia realizar diversas visitas de este tipo; y d) los que viajan por otros motivos pero que a lo largo de su estancia pueden consumir más o menos casualmente algún producto cultural en el destino sin tener una motivación especial para ello (Silberberg, 1995: Hughes, 2002).

\section{METODOLOGÍA APLICADA}

Para analizar los tres casos aquí presentados y ver su evolución en estos últimos años, se ha recogido información estadística de los visitantes recibidos en ellos entre los años 2003 y 2017, facilitada por el Departamento de Cultura de la Generalitat de Catalunya (Generalitat de Catalunya 2003; 2009; 2017).

Asimismo, se han realizado 189 encuestas personales al azar entre los visitantes que salían de estas instalaciones, mayores de edad y sin distinción de género. Para ello se contó con la colaboración de miembros del grupo de investigación TUDISTAR, del Departamento de Geografía de la Universitat Autònoma de Barcelona (UAB). Dichas encuestas fueron realizadas a lo largo de varios fines de semana entre los meses de agosto de 2018 y febrero de 2019. En concreto, se han encuestado 62 personas en la cartuja de Scala Dei, 74 en el monasterio de 
Santa María de Vilabertrán y 53 en el monasterio-castillo de Sant Miquel d’Escornalbou, de los cuales una tercera parte fueron encuestados en la temporada de verano, otra tercer parte en la de otoño y la restante tercera parte en la de invierno, con el objetivo de evitar diferencias significativas entre ellos según la época del año en que realizasen la visita.

A los encuestados, después de conocer sus datos socio-demográficos más significativos (género - edad - lugar de residencia habitual - motivo principal del viaje), siguiendo a Scott, Baggio y Cooper (2008) se les preguntó sobre cuál fue la principal forma para obtener la información previa a la visita, ofreciéndoles la posibilidad de escoger entre: las web de internet, las relaciones personales (amigos, parientes, conocidos, compañeros de trabajo etc.), las guías y libros de turismo, las diferentes revistas, las agencias de viajes y oficinas de turismo u otras formas aquí no consideradas.

También, siguiendo a Hall, Gossing y Scott (2015), se les preguntó sobre los factores que más habían apreciado en la visita, proponiéndoles cinco (restauración del edificio - monumentalidad del conjunto - entorno - arquitectura - historia), a valorar entre 1 (muy poco) y 7 (mucho). Siguiendo a estos mismos autores, y con la misma valoración, se quiso conocer cuál era su grado de satisfacción global alcanzado con la visita realizada; y lo mismo con la accesibilidad al recinto y dentro del recinto, con los servicios allí disponibles y con la información recibida, sea en persona o mediante audífonos-guía, folletos o paneles explicativos. Asimismo, se les preguntó sobre el número de visitas ya realizadas anteriormente por ellos al mismo lugar y también sobre cuál fue el motivo principal para realizar el viaje (ver este monumento, otro cercanos, conocer mejor este destino o, simplemente, pasar un día de ocio cultural).

Finalmente, se les preguntó si la visita había cumplido con sus expectativas iniciales previas (con una valoración de: muy alto - alto - normal - flojo - muy flojo) y si recomendarían la visita a otras personas ( $\mathrm{si}-\mathrm{no}$ ).

En cuanto al mapa de situación de los tres conjuntos monumentales aquí analizados se ha utilizado el programa de libre acceso gvSIG, mientras que para los cálculos y gráficos se ha servido del programa Excel de Microsoft.

\section{CASOS CONSIDERADOS}

A continuación se presenta seguidamente un resumen muy sintético de cada uno de los tres complejos monumentales aquí analizados, siendo comunes su inclusión dentro del plan de protección del patrimonio del departamento de Cultura de la Generalitat de Catalunya (rehabilitación - promoción - gestión - mantenimiento), su ámbito religioso, la fecha de inicio de la construcción y su abandono en 1835 debido a la desamortización de Mendizábal. Los tres quedaron en estado ruinoso y fueron, finalmente, rescatados por la Generalitat de Catalunya, siendo rehabilitados y reacondicionados para su puesta en valor turístico y como enseña patrimonial de la comunidad local. 
El monasterio cartujo de Scala Dei se encuentra situado a los pies de la Sierra del Montsant, en las cercanías de La Morera de Montsant y perteneciente, en la actualidad, a la comarca tarraconense del Priorat, famosa por sus vinos. Se trata de un complejo religioso iniciado a finales del siglo XII, siendo la primera cartuja construida en la Península Ibérica. Su época de máximo esplendor fue en el siglo XVIII, con tres claustros y treinta celdas, además de poseer una famosa bodega y realizar un extenso cultivo de la vid en sus alrededores. En 1835 sufrió la desamortización de Mendizábal, por lo que fue abandonada y quedando, con el tiempo, en estado muy ruinoso. Al cabo de unos años pasó a ser una propiedad privada, hasta que, en 1990, fue vendida a la Generalitat de Catalunya, que la está restaurando progresivamente, aunque ya la ha abierto al público, parcialmente, desde 1993. (Fonts, 2004).

El monasterio de Santa María de Vilabertrán está en la población del mismo nombre, en la comarca gerundense del Alt Empordà. Se tiene constancia de que en el siglo XI se instaló allí una comunidad de canónigos, que, con el tiempo, siguieron las normas de San Agustín, bajo las órdenes de un abad. En 1592 fue secularizado y se convirtió en una casa presbiteral. Posteriormente, en 1794 fue saqueado por las tropas de Napoleón y en 1835 sufrió la desamortización de Mendizábal, por lo que fue abandonado. Al finalizar la Guerra Civil española, en 1939, se convirtió en cuartel militar, sufriendo, con el tiempo, un importante deterioro, hasta que, en 1980, fue adquirida por la Generalitat de Catalunya, que rehabilitó los edificios y, además de abrirlo al público, celebra allí un festival anual de música clásica. (Pladevall, 1970; Marqués, 1993).

De la fortaleza romana de Escornalbou, situada cerca de Riudecanyes, en la comarca tarraconense del Baix Camp, ya se tiene constancia en el siglo III, aunque el monasterio anexo no empezó a construirse hasta finales del siglo XII. En 1580 pasó a ser un seminario de los frailes franciscanos. Allí residía una comunidad de monjes, bajo las órdenes de un prior, y otra de canónigos, con una cofradía, aunque pronto pasó a depender del arzobispado de Tarragona.

En 1835 también sufrió la desamortización de Mendizábal, siendo abandonado y quedando en estado bastante ruinoso, hasta que fue vendido. Entonces fue utilizado, progresivamente, como almacén de una explotación minera, corral de ganado y fábrica de monedas falsas. En 1920, un nuevo propietario inició su rehabilitación en profundidad, aunque siguiendo sus propios cánones arquitectónicos, lejanos a la realidad anterior de los edificios. En 1979 fue adquirido, a partes iguales, por la Generalitat de Catalunya y la Diputación de Tarragona, finalizando su restauración y abriéndolo al público. (Pladevall, 1970).

\section{RESULTADOS}

El perfil socio-demográfico del personal encuestado es el que se refleja en el cuadro siguiente (Tabla 1). 
Tabla 1. Perfil socio-demográfico de los encuestados

\begin{tabular}{|c|c|c|c|}
\hline & $\begin{array}{c}\text { Cartuja de } \\
\text { Scala Dei }\end{array}$ & $\begin{array}{l}\text { Santa Ma de } \\
\text { Vilabertrán }\end{array}$ & $\begin{array}{c}\text { Sant Miquel } \\
\text { d'Escornalbou }\end{array}$ \\
\hline $\mathrm{n}$ - encuestas & 62 & 74 & 53 \\
\hline edad media & 50,3 & 46,7 & 43,8 \\
\hline \multicolumn{4}{|l|}{ lugar de residencia habitual } \\
\hline Catalunya & $61 \%$ & $46 \%$ & $64 \%$ \\
\hline resto de España & $35 \%$ & $24 \%$ & $32 \%$ \\
\hline extranjero & $4 \%$ & $30 \%$ & $4 \%$ \\
\hline \multicolumn{4}{|l|}{ motivo principal del viaje } \\
\hline ocio cultural & $74 \%$ & $65 \%$ & $82 \%$ \\
\hline visita familiares / amigos & $8 \%$ & $14 \%$ & $6 \%$ \\
\hline trabajo & $4 \%$ & $7 \%$ & $2 \%$ \\
\hline otros & $14 \%$ & $14 \%$ & $10 \%$ \\
\hline \multicolumn{4}{|l|}{ repetición visita } \\
\hline primera vez & $64 \%$ & $58 \%$ & $75 \%$ \\
\hline segunda vez & $31 \%$ & $27 \%$ & $22 \%$ \\
\hline tres o más veces & $5 \%$ & $15 \%$ & $3 \%$ \\
\hline \multicolumn{4}{|l|}{ objetivo fundamental del viaje a este destino } \\
\hline visitar este monumento & $54 \%$ & $42 \%$ & $58 \%$ \\
\hline ver otros monumentos cercanos & $8 \%$ & $15 \%$ & $4 \%$ \\
\hline pasar el día & $25 \%$ & $28 \%$ & $32 \%$ \\
\hline conocer mejor el territorio & $13 \%$ & $15 \%$ & $6 \%$ \\
\hline
\end{tabular}

Elaboración propia.

Así pues, según los resultados de la encuesta, puede apreciarse que la edad media de los encuestados y encuestadas rondaba los 46 años, siendo mayoritariamente Catalunya su lugar de residencia habitual, mientras que el porcentaje de extranjeros que visitaron estas instalaciones era mínimo, con la excepción de Vilabertrán, debido a la influencia del turismo francés, ya que se encuentra en las proximidades de la frontera de La Junquera, y, además, la cantidad de franceses que veranean en el Empordà es muy apreciable.

El motivo principal del viaje es, en unas tres cuartas partes de los casos encuestados, el ocio cultural; aunque en ocasiones se aprovecha la visita a otros lugares más o menos próximos, por otros motivos, para hacer este desplazamiento. Este fenómeno se pone claramente de manifiesto en los resultados de las respuestas a la pregunta sobre el objetivo fundamental del viaje a este destino, ya que alrededor de la mitad de los encuestados especificaron que la visita a este monumento concreto era su principal objetivo, y cerca del $70 \%$ manifestaron que querían consumir ocio cultural (visitas a éste y/u otros monumentos y conocer mejor el territorio). 
Finalmente, más de un 30\% de los encuestados en el caso de Scala Dei y Vilabertrán manifestaron que estaban repitiendo la visita, mientras que este fenómeno solamente ocurría en un $25 \%$ en el de Escornalbou. En cuanto al modo de acceso al recinto, todos indicaron que lo realizaron mediante vehículo particular.

En cuanto al flujo de visitantes en cada uno de estos tres monumentos en estos últimos quince años (2003-2017), en el caso de Scala Dei (Gráfico 1) se ha mantenido un crecimiento bastante constante (tiene un $\mathrm{R}^{2}$ de 0,7717), pasando de 17.699 en el año 2003 a 33.020 en 2017. Sin embargo, analizándolo más detalladamente, puede observarse una cierta estabilidad hasta el año 2012 (alrededor de 20.000 visitas anuales), con un salto cuantitativo importante en el 2013, cuando pasó de recibir 21.230 visitas a 30.570. Por otra parte, puede comprobarse que la crisis económica iniciada en el 2007 no ha tenido incidencias demasiado significativas en el flujo de visitantes recibidos, ya que hasta el año 2013 se mantuvo la afluencia de público, siguiendo bastante similares comportamientos a los de antes de la crisis.

Gráfico 1. Visitantes de la cartuja de Scala Dei (2003-2017)

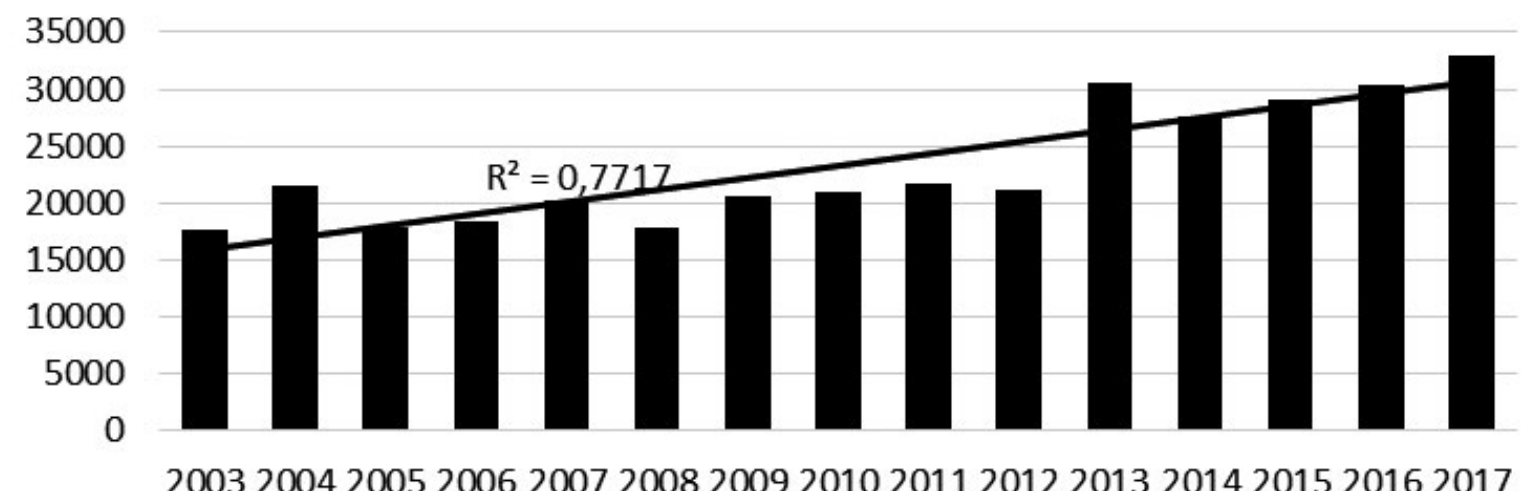

Fuente: elaboración propia a partir de datos facilitados por el Departamento de Cultura de la Generalitat de Catalunya (2018).

Por el contrario, las visitas al monasterio de Vilabertrán han seguido unas pautas muy distintas en este período, tal como puede apreciarse en el Gráfico 2, cuya $\mathrm{R}^{2}$ es solamente de 0,0989 . En el gráfico también puede observarse como en los años inmediatamente anteriores al inicio de la crisis la afluencia de visitantes era decreciente, aumentando considerablemente en los años más álgidos de la recesión económica, pasando de 9.087 en el 2008 a 14.755 en el 2009, aunque volviendo a los niveles anteriores a partir del año 2013, aunque con un cierto repunte en el 2017 (13.638 visitas). 
Gráfico 2. Visitantes del monasterio de Vilabertrán (2003-2017)

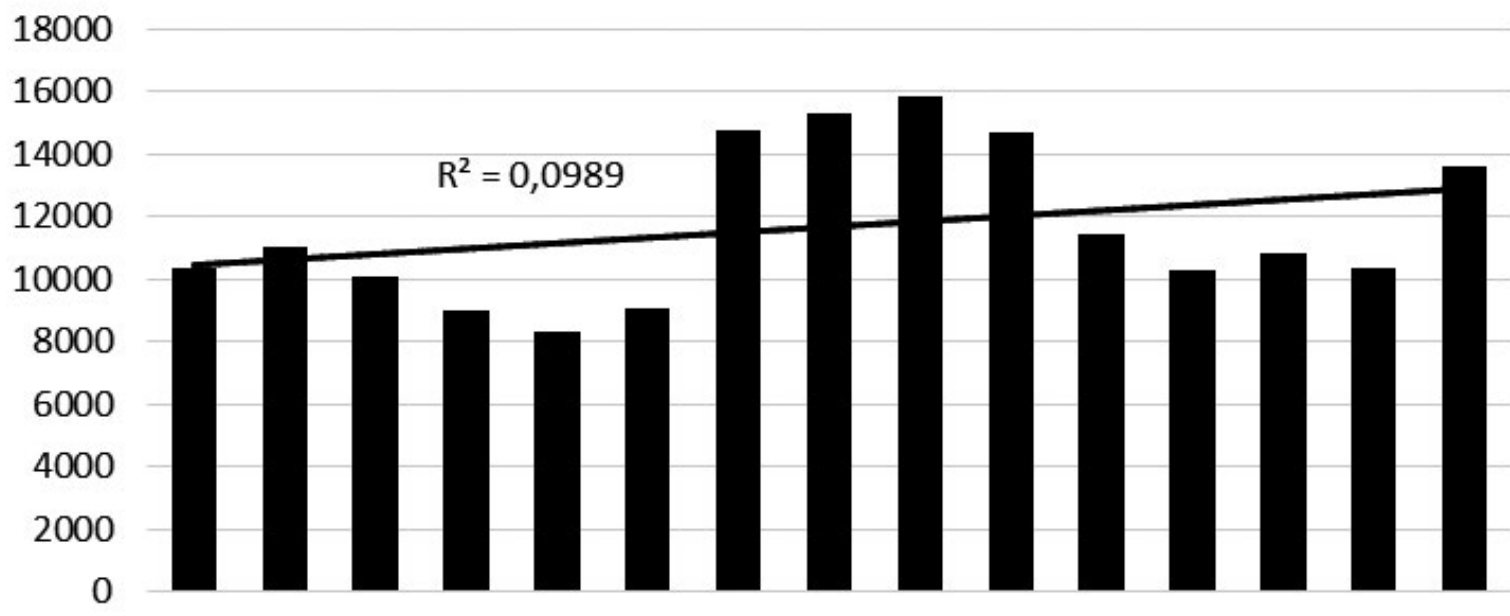

200320042005200620072008200920102011201220132014201520162017

Fuente: elaboración propia a partir de datos facilitados por el Departamento de Cultura de la Generalitat de Catalunya (2018).

En cuanto al monasterio-fortaleza de Escornalbou, la tendencia a lo largo de estos quince años ha sido un flujo decreciente de visitantes (siendo su $\mathrm{R}^{2}$ de 0,3005 ), tal como puede apreciarse en el Gráfico 3, pasando de los 28.947 del año 2003 a los 15.195 del 2008. También puede verse como una vez superado el inicio de la crisis se ha producido un cierto repunte de las visitas, llegando hasta las 20.020 en el 2017, aunque lejos de las casi 30.000 que había al inicio del período.

Gráfico 3. Visitantes del monasterio-fortaleza de Escornalbou (2003-2017)

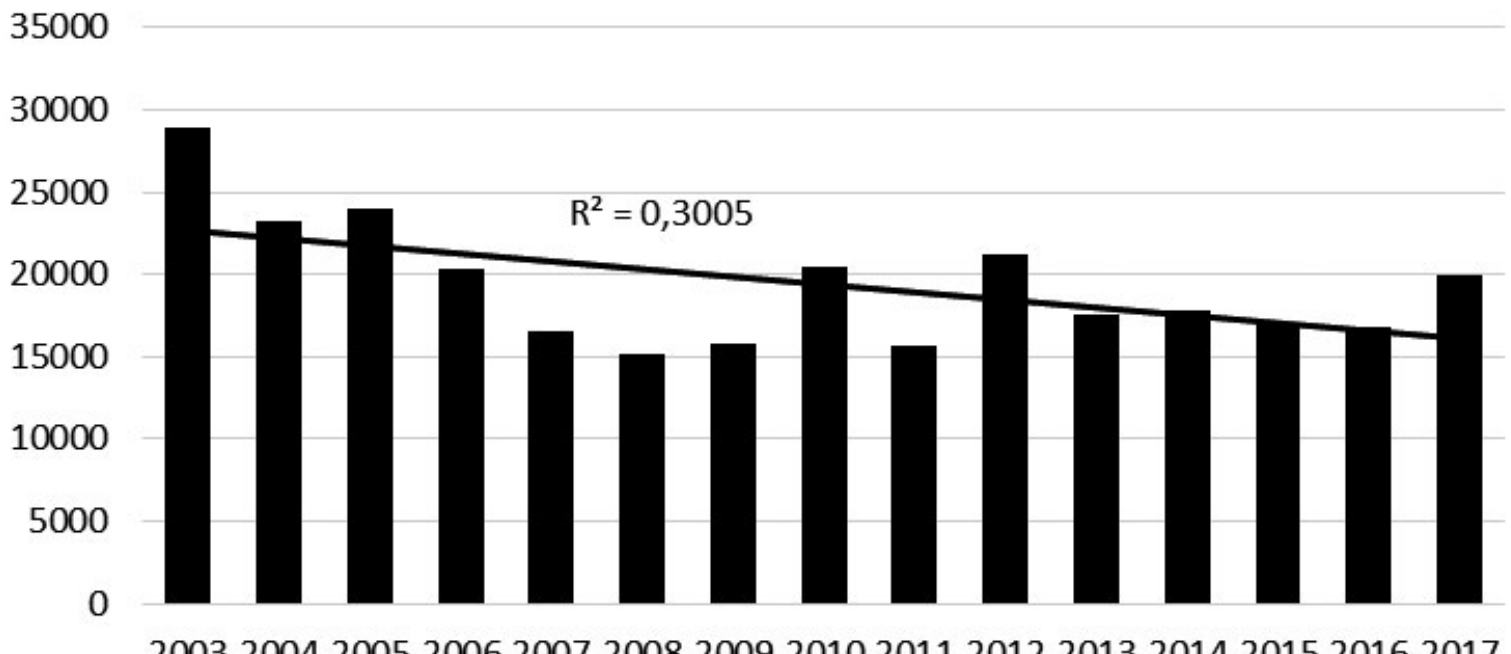

Fuente: elaboración propia a partir de datos facilitados por el Departamento de Cultura de la Generalitat de Catalunya (2018). 
Comparando la evolución de los visitantes recibidos en estos tres conjuntos monumentales a lo largo del período aquí considerado (Gráfico 4), puede apreciarse que, en conjunto, los tres casos siguen distintas tendencias.

Gráfico 4. Comparación de la evolución de los visitantes en los tres casos considerados (2003-2017)

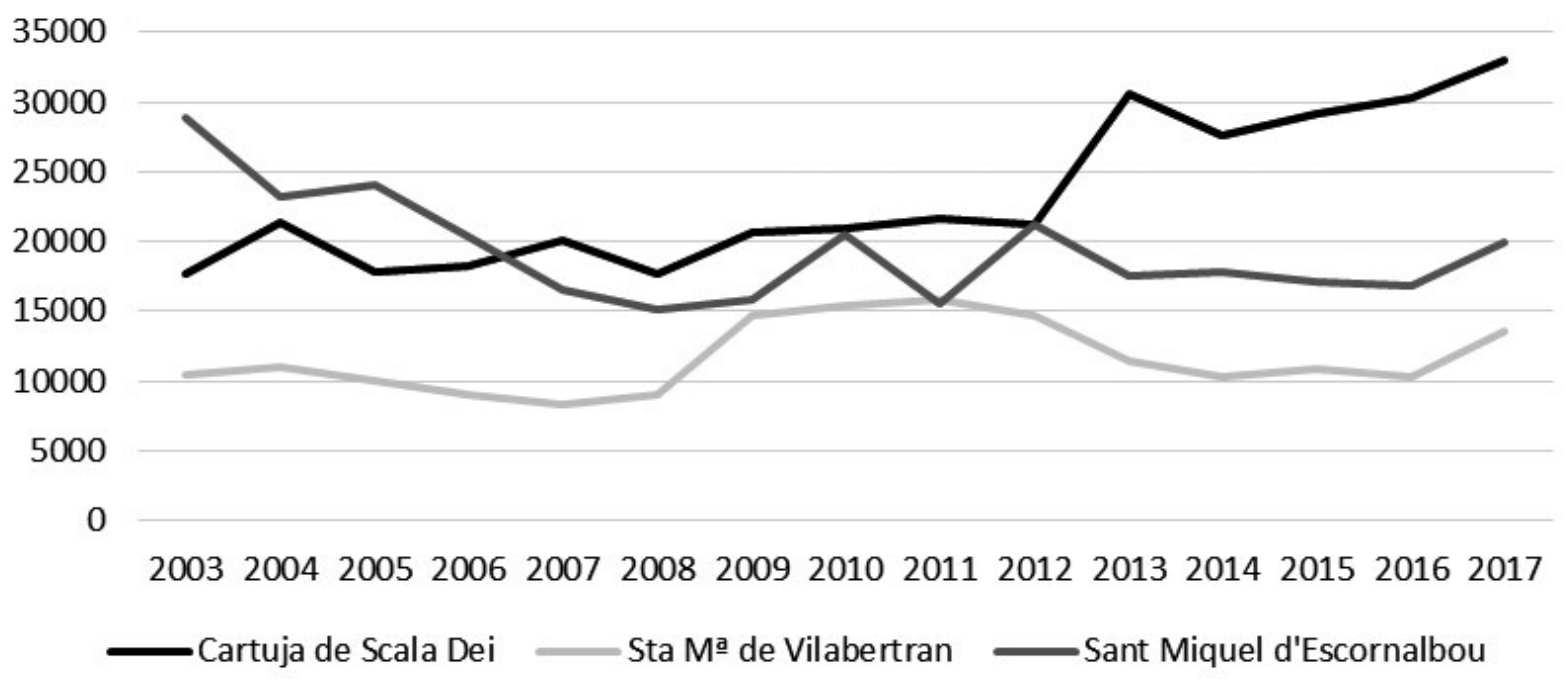

Fuente: elaboración propia a partir de datos facilitados por el Departamento de Cultura de la Generalitat de Catalunya (2018).

Sin embargo, si nos fijamos solamente en los años anteriores al inicio de la crisis (el período 2003-2007) podemos apreciar como las tendencias en los flujos de visitantes recibidos en Scala Dei y Vilabertrán son similares, manteniéndose dicha similitud hasta el año 2012, cuando la similitud pasa a ser entre Vilabertrán y Escornalbou. Por otra parte, la diferencia tan significativa que existe con relación a los visitantes recibidos en los años más álgidos de la crisis puede deberse a que Vilabertrán está muy cerca de la Costa Brava y de la frontera francesa, por lo que el porcentaje de visitantes potenciales del monasterio ampurdanés es mucho más acusado que en el caso de la cartuja o el monasterio-fortaleza tarraconenses.

No obstante estas diferencias, si se observa todo el período considerado puede afirmarse que la crisis económica no ha incidido de manera demasiado decisiva en la evolución de las visitas recibidas en estos tres elementos monumentales, manifestándose un cierto estancamiento en Vilabertrán y Escornalbou, mientras que en Scala Dei la tendencia es más positiva. A pesar de ello, en los tres casos puede verse como en el año 2017 se ha producido un repunte de las visitas recibidas.

Una vez realizado el análisis descriptivo de las cantidades de visitantes recibidos anualmente, entre 2003 y 2017, en los tres casos aquí considerados, vamos a analizar los resultados de las encuestas. Así, respecto a la pregunta relativa al modo de obtención de la información 
previa a la realización de la visita (Tabla 2), los encuestados manifestaron una cierta divergencia, en función del destino. Aquellos que fueron a Vilabertrán utilizaron principalmente las web de internet para informarse previamente, mientras que los que se desplazaron a Scala Dei o a Escornalbou tuvieron las relaciones personales como fuente primigenia de información. Asimismo, la información facilitada en las agencias de viajes o en las oficinas de información turística solamente fueron consideradas entre los visitantes de Vilabertrán, los cuales también utilizaron más, para informarse antes del viaje, las guías y libros de turismo, así como las revistas.

Tabla 2. Modo de obtención de información previa a la visita

\begin{tabular}{|c|c|c|c|}
\hline & $\begin{array}{c}\text { Cartuja de Scala } \\
\text { Dei }\end{array}$ & $\begin{array}{l}\text { Santa Ma de } \\
\text { Vilabertrán }\end{array}$ & $\begin{array}{c}\text { Sant Miquel } \\
\text { d'Escornalbou }\end{array}$ \\
\hline internet & $28 \%$ & $40 \%$ & $27 \%$ \\
\hline relaciones personales & $55 \%$ & $21 \%$ & $64 \%$ \\
\hline $\begin{array}{r}\text { agencia viajes y } \\
\text { oficinas de turismo }\end{array}$ & $0 \%$ & $6 \%$ & $0 \%$ \\
\hline $\begin{array}{r}\text { guías y libros de } \\
\text { turismo }\end{array}$ & $5 \%$ & $9 \%$ & $4 \%$ \\
\hline revistas & $8 \%$ & $12 \%$ & $5 \%$ \\
\hline otros & $4 \%$ & $12 \%$ & $1 \%$ \\
\hline
\end{tabular}

Fuente: elaboración propia a partir de los resultados de las encuestas.

Con relación a la pregunta relativa a los factores que más han apreciado los encuestados una vez realizada la visita (Gráfico 5), tres de los factores propuestos han sido apreciados por todos. En concreto, son la monumentalidad de conjunto, el entorno y la arquitectura románica y gótica de los edificios. Sin embargo, los otros dos restantes presentan notables discrepancias según cual sea el destino.

Así, la restauración de los edificios es mucho más apreciada por los visitantes de Vilabertrán, seguidos a distancia por los de Escornalbou, mientras que el conocimiento de la historia del lugar es más valorado por los que se han desplazado hasta los monasterios de Scala Dei y Vilabertrán. 
Gráfico 5. Factores más apreciados por los visitantes

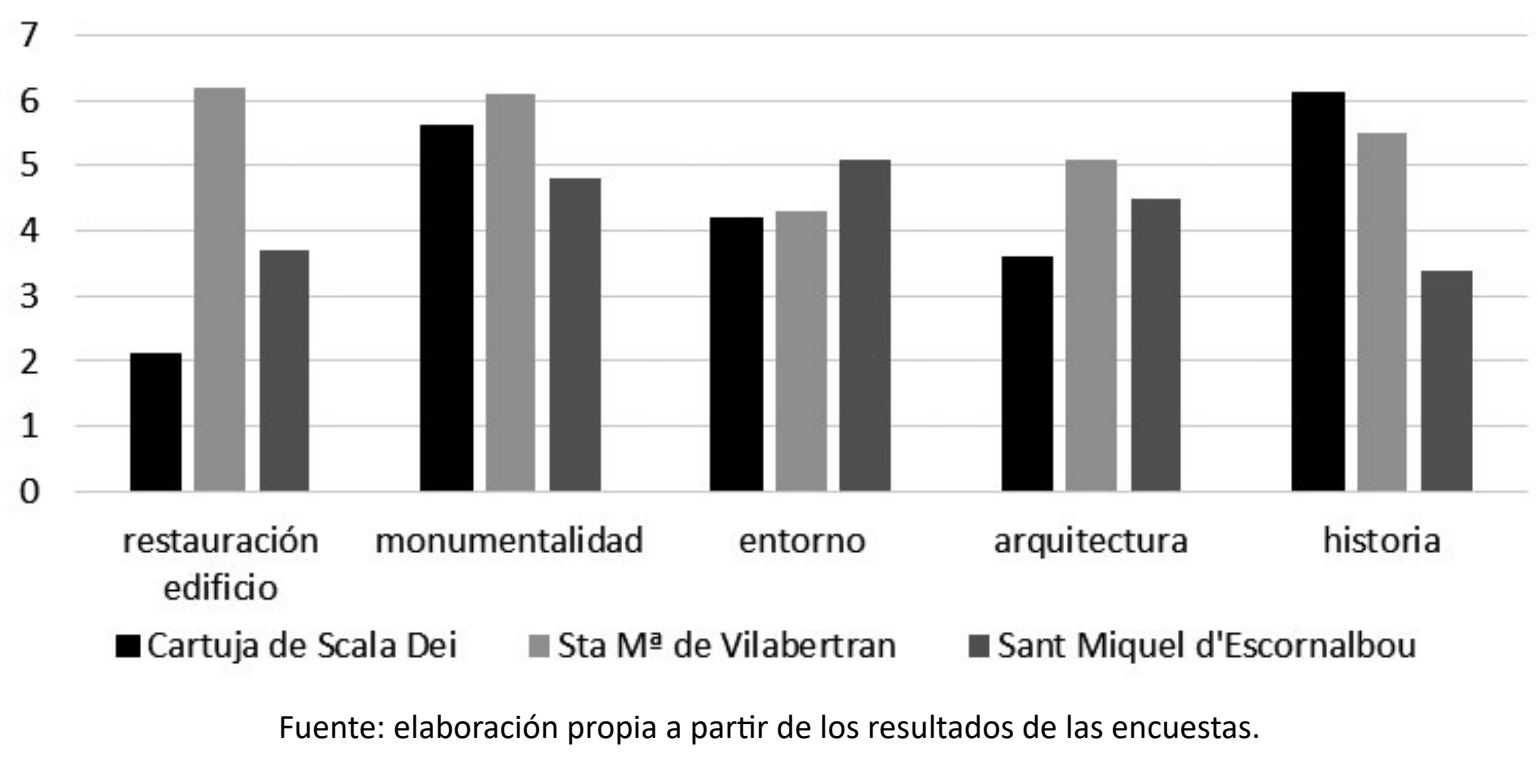

No obstante estas pequeñas diferencias, la mayoría de los visitantes encuestados valoran bastante positivamente todos los factores propuestos. La única excepción ha sido la restauración de la cartuja de Scala Dei. La razón es que todavía está en una fase bastante embrionaria y solamente puede visitarse la iglesia, uno de los claustros y algunas celdas colindantes. Curiosamente, la restauración muy "sui generis" del monasterio-fortaleza de Escornalbou ha sido bastante bien apreciada, a pesar de no seguir los patrones arquitectónicos reales del edificio.

Con respecto al grado de satisfacción obtenido con la visita (Gráfico 6), en global ha sido muy positivo en todos los tres casos, superando la valoración 4 (sobre 7), aunque con un ligero predominio en Vilabertrán.

Gráfico 6. Grado de satisfacción con la visita

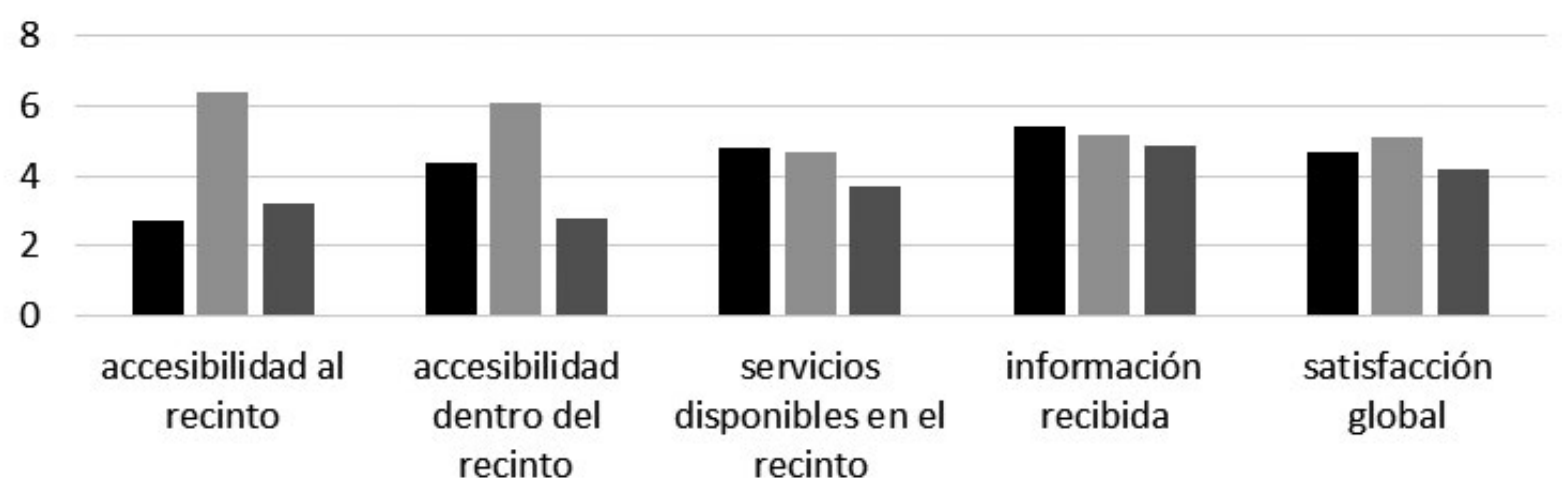

- Cartuja de Scala Dei $\quad$ Sta Maㅡ de Vilabertran $\quad$ Sant Miquel d'Escornalbou

Fuente: elaboración propia a partir de los resultados de las encuestas. 
Ello se corresponde con los resultados de la pregunta referente al grado de cumplimiento de las expectativas iniciales (Gráfico 7), donde más del $70 \%$ de los encuestados manifestaron que dicho cumplimiento fue alto o muy alto y que el $85 \%$ o más recomendarían la visita a otras personas en los casos de Scala Dei y Vilabertrán, mientras que en Escornalbou el cumplimiento de las expectativas iniciales fue solamente del $46 \%$, aunque el $76 \%$ sí que recomendaría la visita.

Gráfico 7. Grado de cumplimiento de la motivación inicial y nivel de recomendación de la visita

\begin{tabular}{|c|c|c|c|}
\hline & $\begin{array}{l}\text { Cartuja de } \\
\text { Scala Dei }\end{array}$ & $\begin{array}{l}\text { Santa Ma de } \\
\text { Vilabertrán }\end{array}$ & $\begin{array}{l}\text { Sant Miquel } \\
\text { d'Escornalbou }\end{array}$ \\
\hline \multicolumn{4}{|l|}{$\begin{array}{l}\text { grado de cumplimiento de las } \\
\text { expectativas iniciales de la visita }\end{array}$} \\
\hline muy alto & $32 \%$ & $25 \%$ & $19 \%$ \\
\hline alto & $40 \%$ & $48 \%$ & $27 \%$ \\
\hline normal & $16 \%$ & $20 \%$ & $33 \%$ \\
\hline flojo & $8 \%$ & $5 \%$ & $15 \%$ \\
\hline muy flojo & $4 \%$ & $2 \%$ & $6 \%$ \\
\hline \multicolumn{4}{|l|}{ recomendación de la visita } \\
\hline si & $85 \%$ & $89 \%$ & $76 \%$ \\
\hline no & $15 \%$ & $11 \%$ & $24 \%$ \\
\hline
\end{tabular}

Fuente: elaboración propia a partir de los resultados de las encuestas.

En el resto de las preguntas planteadas en la encuesta (accesibilidad al y dentro del recinto; servicios disponibles en el recinto; información recibida durante la visita) la valoración dada por los encuestados también ha sido bastante positiva, especialmente en el caso de Vilabertrán. Las únicas excepciones han sido la accesibilidad al recinto en Scala Dei y Escornalbou, y la accesibilidad dentro del recinto en Escornalbou.

Finalmente, respecto al número de veces que habían visitado este mismo lugar (Tabla 2), un apreciable porcentaje de los encuestados repetía la visita (el 36\% en Scala Dei, el $42 \%$ en Vilabertrán y el $25 \%$ en Escornalbou), aunque la mayoría manifestaron que era la primera vez que lo hacían (algo más de la mitad en el caso de Vilabertrán, dos terceras partes en Scala Dei y tres cuartas partes en Escornalbou). Sin embargo, un 15\% respondió que ésta ya era su tercera o más visita a Vilabertrán. Ello concuerda con su situación muy próxima a la Costa Brava y a la frontera francesa, así como su gran facilidad de acceso desde la autopista AP-7.

Asimismo, vemos que existe una correlación entre el grado de satisfacción global con la visita y el porcentaje de repetición, ya que cuanto mayor el dicho índice, más veces se repite la visita al mismo lugar. En efecto, los visitantes encuestados en Vilabertrán manifestaron un grado de satisfacción con la visita de 5,1 (sobre 7) y un $42 \%$ de repeticiones, mientras que los que estaban en Scala Dei dieron un 4,7 y un porcentaje del $36 \%$ y los de Escornalbou un 4,2 y un $25 \%$. 
Tabla 3. Grado de repetición de la visita

\begin{tabular}{|r|c|c|c|}
\hline repetición visita & $\begin{array}{c}\text { Cartuja de } \\
\text { Scala Dei }\end{array}$ & $\begin{array}{c}\text { Santa Ma de } \\
\text { Vilabertrán }\end{array}$ & $\begin{array}{c}\text { Sant Miquel } \\
\text { d'Escornalbou }\end{array}$ \\
\hline primera vez & $64 \%$ & $58 \%$ & $75 \%$ \\
\hline segunda vez & $31 \%$ & $27 \%$ & $22 \%$ \\
\hline tres o más veces & $5 \%$ & $15 \%$ & $3 \%$ \\
\hline
\end{tabular}

Fuente: elaboración propia a partir de los resultados de las encuestas.

\section{CONCLUSIONES}

En este artículo se ha analizado la evolución de los visitantes recibidos en estos últimos quince años en tres conjuntos monumentales románico-góticos situados en Catalunya, que se encontraban en estado ruinoso debido a la desamortización de Mendizábal y que han sido restaurados, promocionados y gestionados por la Generalitat de Catalunya y puestos en valor turístico, con el objetivo de dar a conocer la historia y la arquitectura de una parte importante de la historia y la cultura del país, tanto para la población autóctona como foránea. Dicho reacondicionamiento también ha servido para promocionar y ayudar a impulsar al desarrollo económico, directo e indirecto, de unos territorios de interior.

También se ha podido comprobar como la crisis económica iniciada en el año 2007 no ha afectado demasiado al flujo de visitantes recibidos anualmente en estos tres casos, aunque tampoco se ha producido un crecimiento espectacular de visitantes, siguiendo cada caso una dinámica evolutiva distinta al resto. En el caso de Scala Dei la tendencia ha sido creciente, en el de Escornalbou decreciente y en el de Vilabertrán oscilante. Por otra parte, los visitantes, cada vez más experimentados y exigentes, se han informado previamente de forma mayoritaria sobre lo que van a visitar, utilizando para ello preferentemente las herramientas disponibles en internet en el caso de Vilabertrán, y las relaciones personales en los otros dos casos.

No obstante, se ha constatado que nos encontramos ante un turismo de proximidad, ya que la mayoría de los visitantes en los tres casos analizados han manifestado que residen en la propia Catalunya y han realizado el viaje para satisfacer fundamentalmente su motivación de ocio cultural.

Asimismo, las expectativas previas a la visita se han cumplido mayoritariamente, provocando que el grado de satisfacción alcanzado con la visita haya sido bastante alto en todos los casos, ya que se trata de un tipo de turismo, el turismo de ocio cultural, generalmente alejado del turismo de masas, que permite disfrutar de la visita, con tranquilidad y asimilando toda la información de que se dispone, antes y durante el recorrido, permitiéndote, además, la posibilidad de conocer y degustar otros elementos de la cultura local, como, por ejemplo, su gastronomía, su artesanía o su folklore. En definitiva se trata de" reconocer" en lugar de "conocer".

De este modo, se ha podido confirmar que con la restauración y puesta en valor turístico del patrimonio cultural, por una parte, sus consumidores suelen alcanzar un alto grado 
de satisfacción, repitiendo la visita o, al menos, promocionándola, además de efectuar otros gastos adicionales en el territorio visitado. Por la otra, se establece una nueva vía de apoyo al desarrollo económico y socio-cultural de la comunidad local.

Así pues, con estos caso aquí expuestos se ha podido constatar cómo en algunos territorios de interior con un significativo patrimonio cultural, desde las administraciones públicas se han realizado esfuerzos considerables para ponerlo en valor turístico, junto a otras variables complementarias, como, por ejemplo, las infraestructuras y los accesos, los servicios y la seguridad. De esta forma se han satisfecho las ansias de conocimiento de muchas gente que no deseaba limitarse a ver monumentos antiguos sino que también deseaba entender su historia y las relaciones sociales que allí se establecieron.

También se ha podido observar que cada vez es más necesario que estos territorios deben dinamizar sus potencialidades turísticas, canalizándolas hacia el desarrollo de nuevos productos que satisfagan las motivaciones de unos turistas cada vez más experimentados y exigentes, que demandan experiencias y actividades personalizadas y con la mejor relación calidad-precio. Ante esta situación, el desarrollo turístico sostenible se convierte en un proceso integrado donde los aspectos económicos, sociales, culturales y medioambientales se interrelacionan con los agentes que los gestionan. Para ello es importante contar con la cooperación de todos los agentes implicados en estas actividades (comunidad local, administraciones públicas, empresas, asociaciones, instituciones de investigación, turistas y medios de comunicación).

Además, ha podido comprobarse que la valorización turística del patrimonio que se encontraba en fase de desaparición es una alternativa que permite reforzar en los territorios de interior, históricamente alejados del flujo turístico tradicional, el sentimiento identitario y de pertenencia a un lugar, sus tradiciones y su historia. Asimismo, ante el impacto económico de la crisis, se ha convertido en una herramienta que, directa o indirectamente, ha ayudado a crear puestos de trabajo, retener la población y mejorar su renta personal.

Sin embargo, estos nuevos productos turísticos pueden ser efímeros, ya que, por una parte, la competencia en la oferta es cada vez mayor y, por la otra, su debilidad limita la reacción ante los impactos negativos. No puede olvidarse que los turistas son más vulnerables a las situaciones de recesión o, simplemente, de moda o de competencia entre destinos, ya que su elección se basa en las expectativas y en la confianza.

Por ello es necesario que su desarrollo sea sostenible, involucrando a todos los agentes y creando clústeres cooperativos alrededor de un determinado producto, ya que así pueden ayudarse mutuamente con el objetivo de conseguir una mejor competitividad del destino en general. Pero no sólo el clúster debe ser sostenible sino que también, desde la comunidad local y las administraciones públicas, se debe gestionar la resiliencia del destino, ya que la capacidad de transformación es una característica fundamental en los sistemas sustentables sociales, ecológicos, políticos, económicos o tecnológicos.

Finalmente, hay que tener en cuenta que en este trabajo solamente se ha analizado una muestra relativamente pequeña de visitantes, lo que siempre puede llevar a conclusiones inexactas, por lo que, para llegar a conclusiones más extrapolables sería necesario realizar 
estudios similares en otros lugares y con mayores tamaños de muestra. Asimismo, para la selección de los indicadores aquí utilizados se ha partido de otros trabajos académicos previos, así como de la experiencia y conocimiento personal de los investigadores que han colaborado en este trabajo, por lo que, sin duda, puede presentarse un importante componente de subjetividad.

\section{BIBLIOGRAFÍA}

Blanco, A. y Genovés, G. (2005): Las tecnologías de la información y la comunicación en el desarrollo del turismo rural. Documents d'Anàlisi Geogràfica, 46: 105-117.

Cànoves, G.; Villarino, M.; Blanco, A., De Uña, E. y Espejo, C. (2014). Turismo de interior: renovarse o morir. Valencia: Servei de Publicacions de la Universitat de Valencia.

Di Meo, G. (1995). Patrimoine et territoire. Une parenthèse conceptuelle. Herodote, 78: 15-34. Donaire, J.A. (2012). Turismo cultural. Entre la experiencia y el ritual. Girona: Editorial Vitel.la. Du Cros, H. y McKercher, B. (2015). Cultural Tourism. London: Routledge.

Farrell, B.H. y Twining-Ward, L. (2004). Reconceptualising tourism. Annals of Tourism Research, 31: 274-295.

FlØysand, A. y Jakobsen, S.E. (2007). Commodification of rural places: A narrative of social fields, rural development, and football. Journal of Rural Studies, 23 (2): 206-221.

Fonts, A. (2004). Monografía acerca del origen del monasterio de Scala Dei y de la villa de Poboleda. Barcelona: Miguel Seguí.

García Hernández, M. (2014). Nuevos lugares y nuevos productos turísticos. En: López Palomeque, F. y Cànoves, G. (eds.). Turismo y territorio. Innovación, renovación y desafíos. Valencia: Tirant Humanidades, pp. 333-359.

Generalitat de Catalunya (2003). Estadístiques culturals de Catalunya 2003. Barcelona: Departamento de Cultura de la Generalitat de Catalunya

Generalitat de Catalunya (2009). Estadístiques culturals de Catalunya 2009. Barcelona: Departamento de Cultura de la Generalitat de Catalunya

Generalitat de Catalunya (2017). Estadístiques culturals de Catalunya 2017. Barcelona: Departamento de Cultura de la Generalitat de Catalunya

Goeldner, C.; Ritchie, J. y McIntosh, R. (2000). Tourism, Principles, Practices, Philosophies. New York: John Wiley \& Sons, Inc.

Hall, C.M.; Gossing, S. y Scott, D. (2015). The Routledge Handbook of Tourism and Sustainability. London: Routledge.

Hatch M.J. y Cunliffe, A. L. (2013). Organization Theory, modern, symbolic and postmodern perspectives. Oxford: Oxford University Press.

Hu, J.W. y Ritchie, B. (1993). Measuring Destination Attractiveness. A Contextual Approach. Journal of Travel Research, 32 (2): 25-34.

Hudson, R. (2010). Resilient regions in an uncertain world: wishful thinking or a practical reality? Cambridge Journal of Regions, Economy and Society, 3: 11-25.

Hughes, H.L. (2002). Gay men's holiday destination choice: A case of risk and avoidance. International Journal of Tourism Research, 4 (4): 299-312.

Lyon, D. (1996). La posmodernidad. Madrid: Alianza.

Lyotard, J. F. (2000). La condición posmoderna. Madrid: Cátedra. 
López Palomeque, F. y Cànoves, G. (eds.) (2014). Turismo y territorio. Innovación, renovación y desafíos. Valencia: Tirant Humanidades.

Marqués, J.M. (1993). Canónica de Santa María de Vilabertrán. Barcelona: Curiel Edicions Catalanes.

Martínez, V. (2006). Ocio y turismo en la sociedad actual. Los viajes, el tiempo libre y en entretenimiento en el mundo globalizado. Madrid: MacGraw-Hill.

Martínez, V. (2015). Desarrollo y nuevos retos en el ocio-turismo del siglo XXI. Madrid: Ediciones Académicas Ediasa.

Martos, M. y Pulido, J.I. (2010). Una aproximación al análisisde la actividad turística en la ciudad. Papers, 47-48: 39-57.

McKercher, B. (2002). Towards a classification of cultural tourists. International Journal of Tourism Research, 4: 29-38.

Mínguez, C. (2007). Patrimonio cultural y turismo en los Reales Sitios de la Comunidad de Madrid y sus incidencias en el territorio. Tesis doctoral. Madrid: Universidad Complutense de Madrid. Facultad de Geografía e Historia.

Molina, S. (2006). El posturismo. Turismo y posmodernidad. Mèxico: Editorial Trillas

Pearce, D.G. (2012). Frameworks for Tourism Research. Wallingford: CABI.

Peral. J. (1999). Sociedad. Patrimonio. Modernidad. Consideraciones sobre la contemporaneidad. Legado e intervenciones. Boletín del Instituto Andaluz de Patrimonio Histórico, 28: 110-115.

Pladevall, A. (1970). Els monastirs de Catalunya. Barcelona: Ediciones Destino.

Prat, J.M. (2012). Análisis de la dinámica relacional generada por las websites de Internet en el desarrollo de los turismos marginales. Pasos. Revista de Turismo y Patrimonio Cultural, $10(3), 239-252$.

Prat, J.M. (2013). El turismo industrial como elemento de revalorización del territorio: un análisis desde las relaciones sociales presentes en los destinos. Análisis de casos en Cataluña, Escocia y Alsacia. Tesis doctoral. Bellaterra: Universitat Autònoma de Barcelona.

Prat, J.M. y Cànoves, G. (2014). Análisis de la evolución de la concentración geográfica de los establecimientos de turismo rural en Cataluña. Anales de Geografía de la Universidad Complutense de Madrid, 34 (1): 155-177.

Richards, G. (1996). Cultural Tourism in Europe. Wallingford: CAB Internacional.

Richards, G. y Raymond, C. (2000). Creative Tourism. ATLAS News, 23; 16-20.

Richards, G. (2011). Creativity and tourism: The state of the art. Annals of Tourism Research, 38 (4): 1.225-1.253.

Richards, G. (2017). El consume de turismo en la posmodernidad o en la modernidad líquida. Oikonomics, no 7 .

Rivera, J y Fernández-Baca, R. (2004). Del Patrimonio de las élites culturales al patrimonio de la sociedad democrática. Boletín del Instituto Andaluz del Patrimonio Histórico, 50: 38-39.

Rojek, C. y Urry, J. (1997). Touring cultures: Transformations of travel and theory. London: Psychology Press.

Scott, N.; Baggio, R. y Cooper, C. (2008). Network Analysis and Tourism. From Theory to Practice. Toronto: Channel View Publications.

Silberberg, T. (1995). Cultural tourism: A business opportunity for museums and heritage sites. Tourism Management, 16: 66-69. 
Smith, M. (2015). Issues in Cultural Tourism Studies ( $3^{\text {rd }}$ ed.). London: Routledge.

Smith, M. y Richards, G. (2012). The Routledge Handbook of Cultural Tourism. London: Routledge.

Torres, P. (2015). El escenario posmoderno del turismo cultural. Caso: centro histórico de Mazatlán. Culicán Rosales (México): Universidad Autónoma de Sinaloa. Juan Pablos Editor.

Urry, J. (1990). The Tourist Gaze. London: SAGE.

Wall, G. Y Mathieson, A. (2006). Tourism: Economic, Physical and Social Impacts. Harlow: Prentice Hall.

Walker, B. y Salt, D. (2006). Resilient thinking. Sustaining Ecosystems and people in a changing world. Washington, DC: Island Press.

Williams, S. y Lew, A. (2015). Tourism Geography. Critical understandings of place, space and experience ( $3^{\text {rd }}$ ed.). London: Routledge. 